\title{
Bipolar neutrosophic distance measure in multi-attribute decision making
}

\author{
Chunfang $\mathrm{Liu}^{1}$ \\ ${ }^{1}$ Shenyang Aerospace University
}

August 11, 2020

\begin{abstract}
Bipolar neutrosophic set(BNS) is a generalization of bipolar fuzzy set and neutrosophic set that can describe the uncertain information from both positive and negative perspectives. In this contribution, we study the multi-attribute decision making methods based on the distance measure under the uncertain information which the attribute weights are incompletely known or completely unknown. We first propose the distance measures of the bipolar neutrosophic sets and analyze the properties of the distance measures. Then, based on the bipolar neutrosophic information, we establish the programming models to derive the attribute weights of the alternatives. Furthermore, we give the multi-attribute decision making method using the distance measure under the environment of BNS. At last, we give a practical application and the result shows the reasonable and effective of the proposed method in dealing with decision making problems.
\end{abstract}

\section{Hosted file}

\begin\{CJK\}\{UTF8\}\{gbsn\}\end\{CJK\}\selectlanguage\{english\}the ideal point method.pdf avail- } able at https://authorea.com/users/350295/articles/475164-bipolar-neutrosophic-distancemeasure-in-multi-attribute-decision-making 